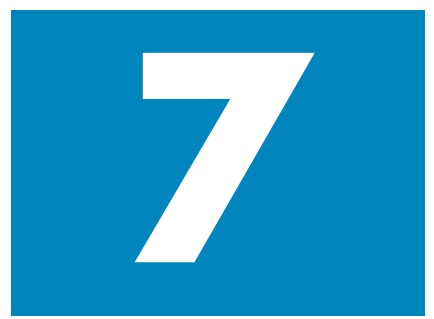

\title{
Basic restorative techniques
}

Leslie Howe, 1 Vincent Barrett, 2 and Paul Palmer, 3

The restorative phase of treatment starts before the implants are placed. It is essential that a clear idea of the final result should be envisaged so that the dentist and patient can appreciate any limitations or compromises that may be needed. The restorative dentist will be responsible for the fabrication of any radiographic or surgical guides that may be required to help in the surgical positioning of the implant. Provisional restorations that maintain appearance and function during implant treatment are also the restorative dentists' responsibility.

The restorative dentist familiar with routine prosthodontic techniques will recognise many of the basic procedures involved in the restorative phase of implant treatment. There is much in common: thorough examination and treatment planning, diagnostic work-up that includes tooth selection, positioning and occlusal contacts, indirect techniques with crown and bridge impression materials, accurate jaw relation and occlusal records. However, implant supported restorations also require a mechanism for attaching the restoration to the implant and this component is termed the abutment. Its selection, placement and the recording of its position with adapted impression techniques is the main difference between conventional and implant prosthodontic techniques.

The standard restorative procedure for implant restorations allows the clinician to record accurate impressions of the abutment by using machine made copings. This is a significant advantage over tooth-borne restorations in that the copings guarantee marginal detail while the impression accurately relates the abutments to one another, any remaining teeth

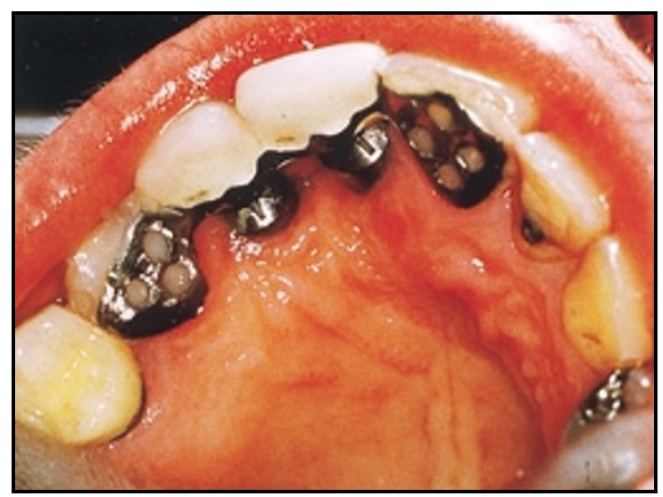

Fig. 1 Rochette bridge used as a provisional restoration

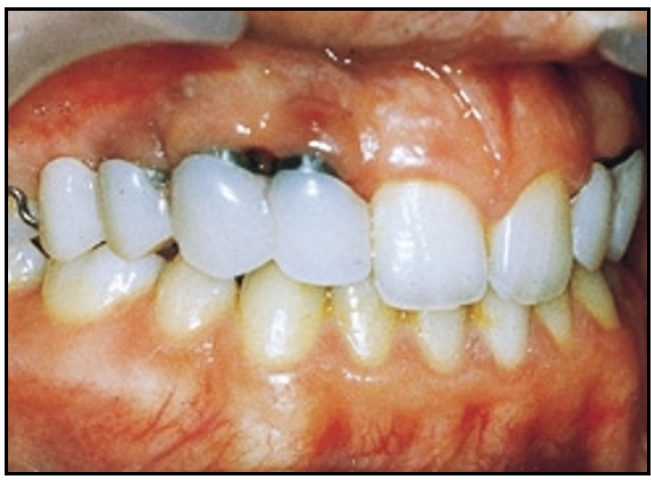

Fig. 2 A provisional partial acrylic denture needs to allow for adjustment when the healing abutments are placed

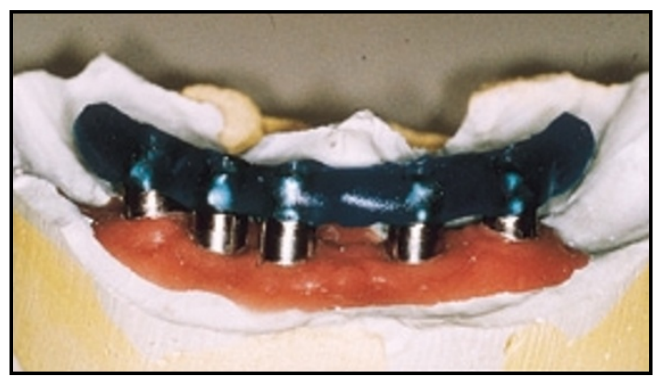

Fig. 3 Standard cylindrical abutments linked together with a composite resin bar ready for try in

and the soft tissue detail. Replicas of the abutments are then placed into the impression which is then cast in dental stone to provide a representation of the abutments on which machine made gold or ceramic cylinders are used to fabricate the restoration.

\section{Provisional restorations}

Before starting implant treatment a provisional or transitional restoration should be made that will be durable throughout what can sometimes be a lengthy course of treatment.

The requirements of a provisional restoration are that it should allow for the implant sites to be minimally loaded immediately after initial surgery. They should also be designed so that they are easily adapted following placement of healing abutments and subsequent implant restorative procedures. In its simplest form a provisional restoration may be a modification to an existing prosthesis but could also involve extensive fixed bridgework made with a metal framework/composite resin veneer.

\section{Some restorative techniques for implant supported restorations will be familiar to dentists used to providing conventional crown and bridgework. The differences and principles involved when using implants are identified.}

In this part, we will discuss:

- Provisional restorations

- Abutment selection

- Seating the abutment

- Impression taking

- Laboratory techniques

- Jaw relation registration

- Try-in appointment

- Framework try-in

- Restoration insertion

- Occlusion

- Follow-up appointment

- Overdentures

${ }^{1}$ Consultant in Restorative Dentistry, Guy's and St Thomas' Hospitals Trust, London SE1 9RT and Specialist in Restorative Dentistry and

Prosthodontics, 21 Wimpole Street, London W1M 7AD; ${ }^{2}$ Private Practitioner, 38 Devonshire Street, London $W 1 ;{ }^{3}$ Clinical Demonstrator in Periodontology, Guy's, Kings and St Thomas' Medical and Dental School, London SE1 9RT and Specialist in Periodontics, 21 Wimpole Street, London W1M 7AD

(C) British Dental Journal 1999; 187: 473-479 


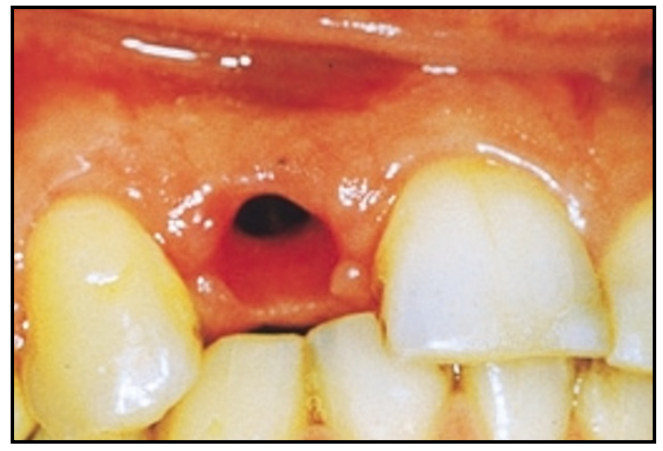

Fig. 4a An Astra single tooth implant with the healing abutment removed

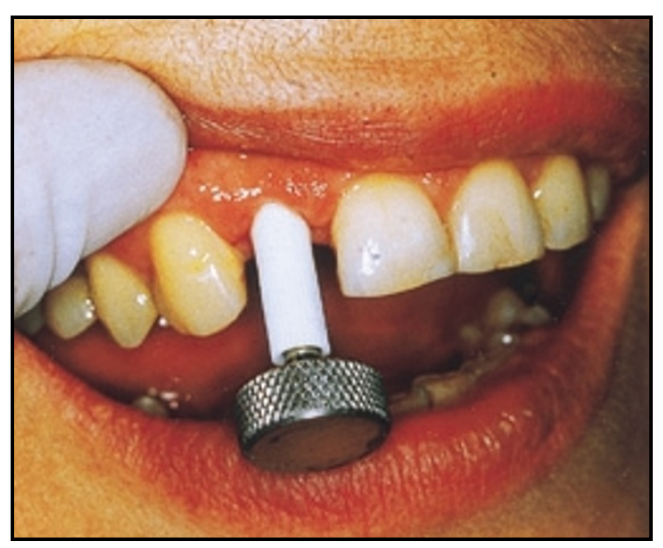

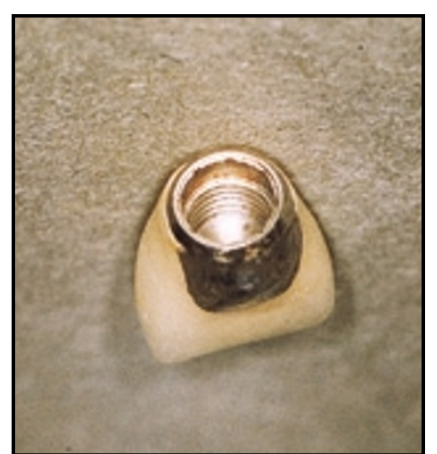

Fig. 4 f Completed crown. Note the retentive shape which cannot rotate
Fig. 4c Abutment seated into implant and abutment screw

tightened

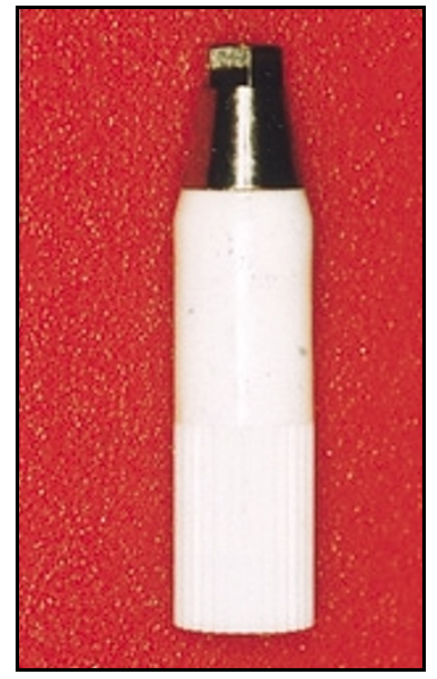

Fig. $4 \mathrm{~b}$ Single tooth abutment attached to holder.

Note the tapered abutment with antirotation shape at the base

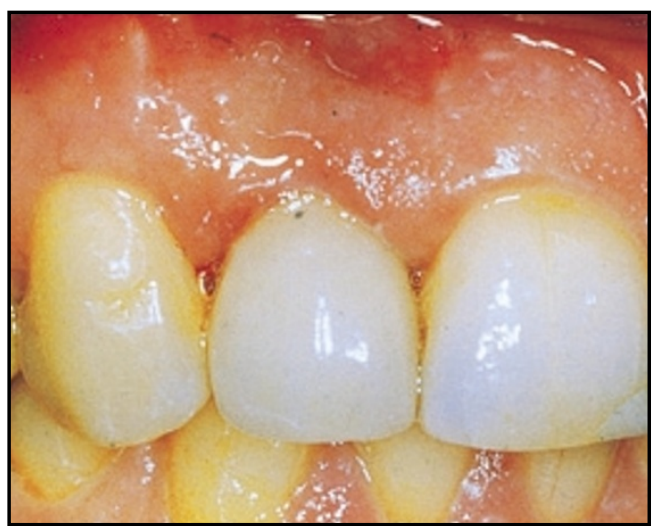

Fig. $\mathbf{4 g}$ Completed crown

For single teeth, consideration should be given to a resin bonded bridge designed so it can be relatively easily removed. A Rochette bridge with a perforated retainer is a good option (Fig. 1). Once again the pontic should be mainly acrylic to allow for later adjustment. Such a bridge can be easily removed and reinserted at the surgical stages, allowing the patient to remain dentate throughout treatment.

When the patient is edentulous, complete dentures should be made to allow adequate relief over the implant sites. Immediately following implant surgery a period of a week or more when dentures cannot be worn is strongly recommended and thereafter soft lining materials such as those for tissue conditioning are used head through into the oral cavity by $1-2 \mathrm{~mm}$.

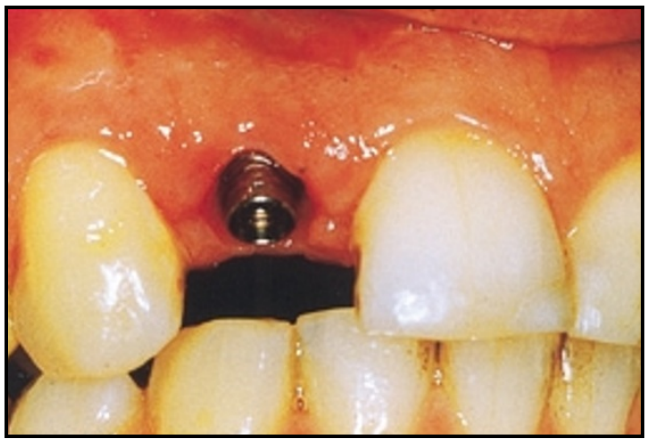

Fig. 4d Single tooth abutment in place

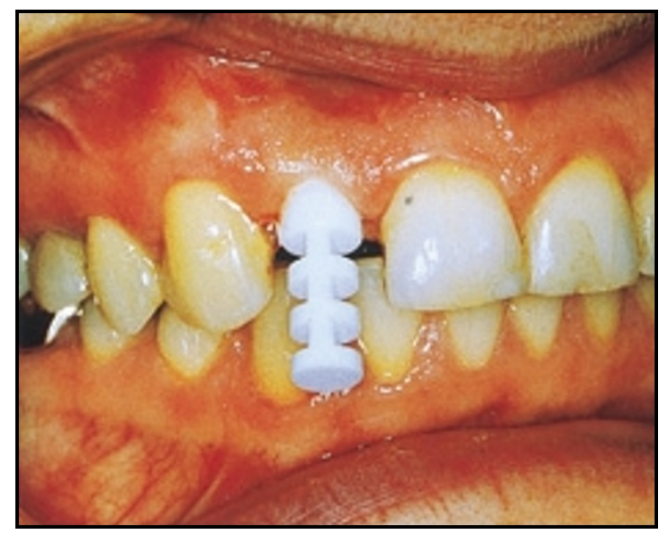

Fig. 4e Impression coping attached

to reline the denture base. The patient should be advised to avoid biting food directly over the surgical site. Removable partial dentures should be constructed with subsequent adjustments in mind which not only include the period following surgical implant placement but also after the healing abutment and final restorative abutment are in position (Fig. 2). If this is done with care a metal base removable partial denture can be made but often a simple acrylic denture is easier to adapt.

Sometimes it is not possible to adapt the provisional restoration after the abutments are placed and consideration should be given to making a provisional restoration on the implant abutments. While this may be seen as an inconvenient further step in treatment, provisional restorations can provide invaluable diagnostic information about tooth arrangement, position and shade for the definitive restoration.

\section{Abutment selection}

Abutments are components that attach to the implant head and are retained to the implant by an abutment screw that extends through the abutment into the body of the implant. The abutment extends through the gingiva into the oral cavity and it provides the support for the restoration.

The simplest abutment is a titanium parallel sided cylinder that extends from the implant 
From the top of this cylinder, bridgework can be made linking the abutments together (Fig. 3). This traditional approach will produce the 'oil rig' style bridge that most readers will be familiar with. It is particularly useful for lower fixed bridges where appearance is not of paramount importance.

In recent years the number of abutments available for all implant systems has dramatically and confusingly increased. The main types are:

\section{Single tooth abutments}

These are designed to incorporate an anti-rotation device both at the junction of the abutment to the implant and also between the abutment and the restoration (Fig. 4). The final restoration can be cemented or screw retained, the cemented restoration being most popular as it is more aesthetic and the angulation of the implant is less important. Single tooth abutments are chosen so that the margin for the restoration is about $2 \mathrm{~mm}$ sub-gingival and machine cylinders for crown construction can be precious metal or porcelain, both having very high fit accuracy.

Fixed bridgework abutments

These abutments are designed to be linked by the restoration to each other and so do not

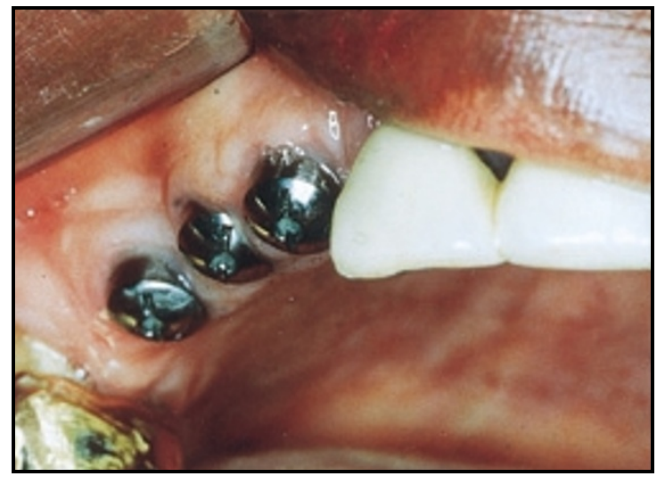

Fig. 5a Three Branemark implants ready to be restored. Healing abutments are in place

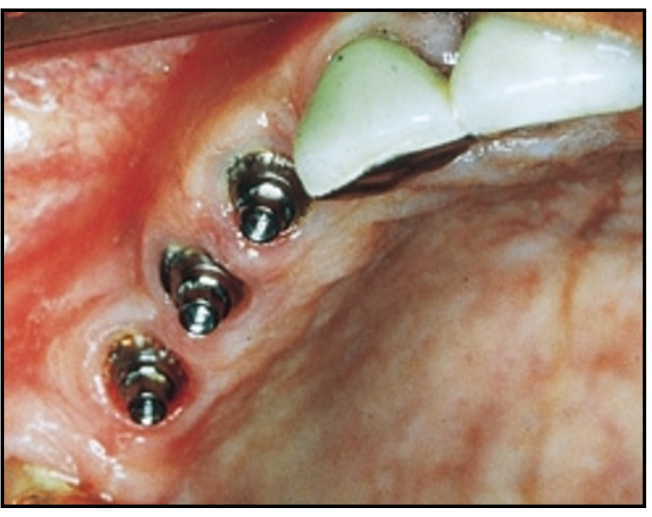

Fig. 5c Bridge abutments in place (Estheticones) require anti-rotation features between the abutment and the bridge (Fig. 5). The abutments are secured to the implant head in the normal way. The bridge end of the abutment is tapered to allow for different paths of insertion of the implants to be overcome by a fixed framework which is either retained by gold screws or conventional cementation. Angled abutments are available to overcome severe alignment problems between implants.

Overdenture abutments

Either ball attachments or magnets can be used which are incorporated into the abutment or several implants can be linked together with a bar onto which a denture can be retained by clips (Fig. 6). Abutments have to be selected dependent upon the available space within the denture.

Armed with the knowledge of which abutment type is to be used an appropriate length of abutment can be chosen. The healing abutment is unscrewed and the height of soft tissue from the rim of the implant head to the gingival margin measured with a periodontal probe. If possible and where necessary, the abutment is selected so that the margin for the restoration is placed $1-2 \mathrm{~mm}$ subgingivally.

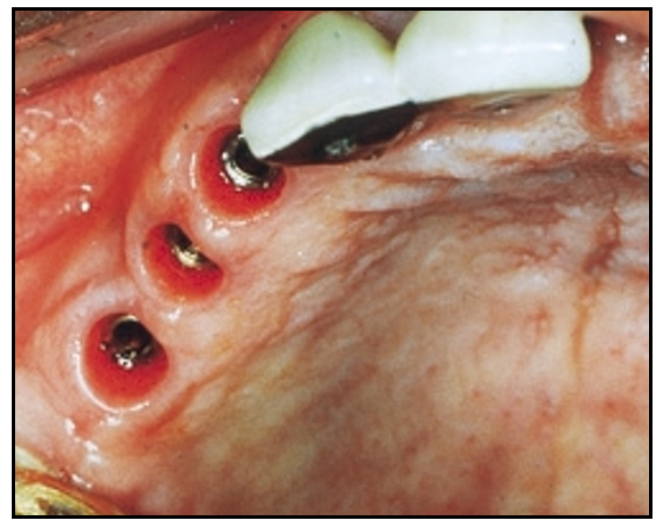

Fig. 5b Healing abutments removed. The head of the implant can be seen with a raised hexagon portion around the screw-hole which will act as positive location for the abutment

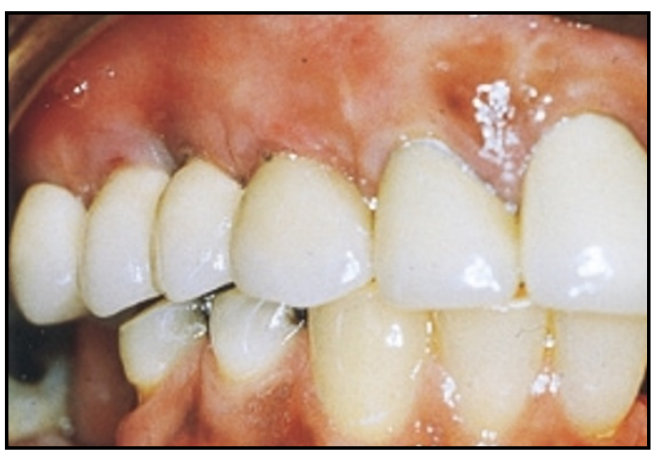

Fig. $5 \mathrm{~d}$ Completed bridge (one extra pontic has been cantilevered distally)

\section{Abutment selection \\ - Single tooth abutments \\ - Fixed bridgework \\ abutments \\ - Overdenture abutments}

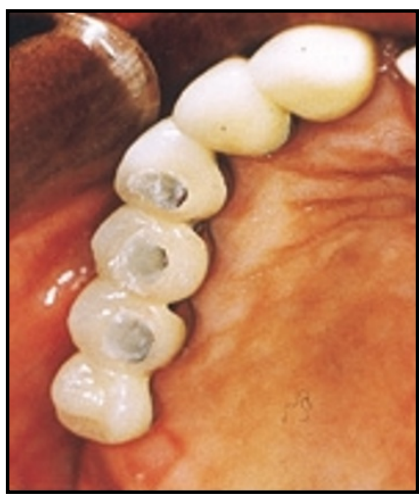

Fig. 5e Palatal view. The access holes for the screws have been restored with composite resin 


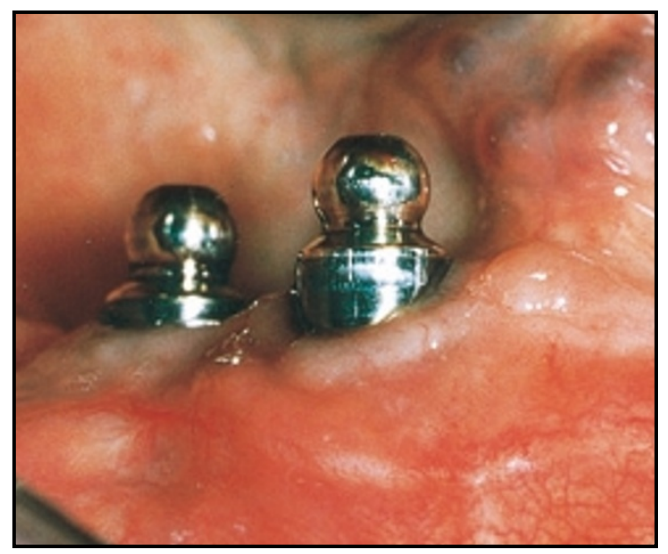

Fig. 6 Overdenture abutments

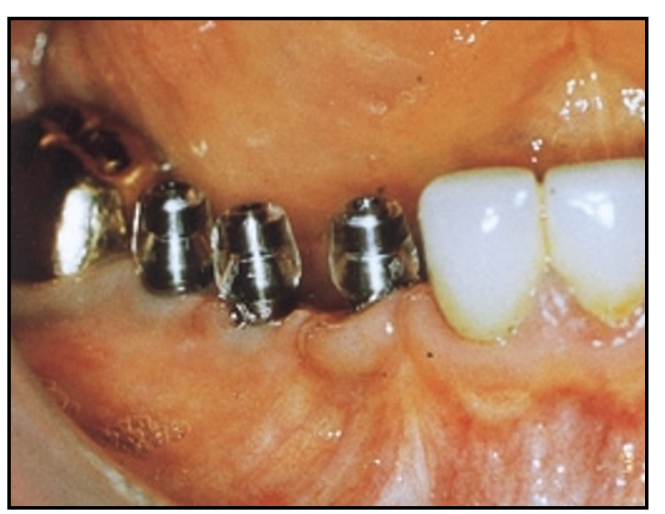

Fig. 8 Astra bridge abutments covered with clear plastic protection copings

\section{Seating the abutment} selected it is then seated onto the implant head. Fig. 7 Radiograph of incorrectly seated single tooth abutment
Once the appropriate abutment has been

provisional restoration will need to be adjusted to accommodate the additional components (Fig. 8). Alternatively the abutment can be removed at the end of each appointment and the healing abutment replaced onto the implant. In this situation final screw tightening is delayed until the final placement of the restoration.

\section{Impression taking}

The aim of an impression for implant restorations is to record the implant positions in a master working cast. Many techniques have been described and materials used in making impressions. While there is no consensus on any one way of making the impression, whichever method is used, it should be done with an understanding of the technique and the properties of the impression material. Impression materials should be resilient enough to be removed from undercuts without distortion and rigid enough to allow for accurate seating of components into the impression and to prevent movement of components during pouring of the impression in dental stone.

Primary impressions can be made with irreversible hydrocolloid (alginate) in a stock tray and for the definitive impression a stock tray

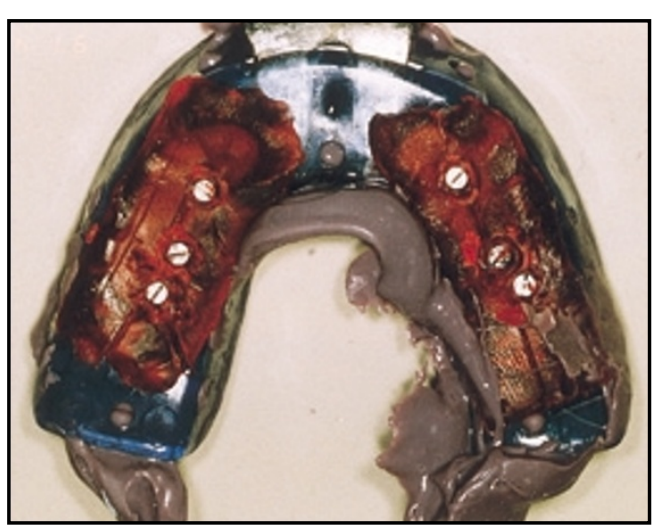

Fig. $9 \mathrm{~b}$ Impression in a stock tray removed from mouth. The retaining screws are visible through the window in the tray to allow them to be

\section{counter-torque device.}

Plastic healing caps are available to cover the abutments once they have been placed and the

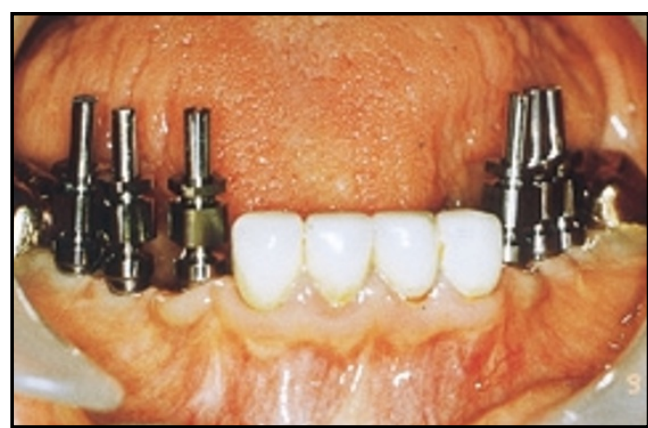

Fig. 9a Impression copings for 'pick up' impression unscrewed

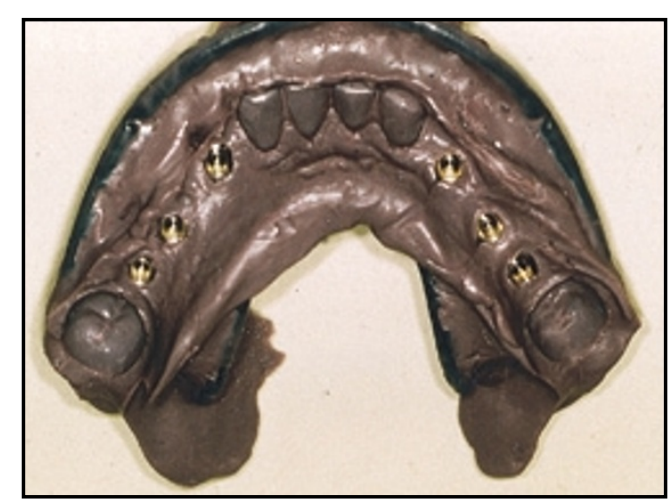

Fig. 9c From the fit surface the impression copings are checked for stability 
$\begin{array}{r}\text { PRACTICE } \\ \text { dental implants } \\ \hline\end{array}$

will be adequate for single tooth implant impressions or short span bridges. For more extensive cases a custom tray is preferable for the final impression, not only to ensure an optimal thickness of impression material for dimensional stability, but also to record sulci and retromolar pad areas.

There are many choices of impression technique. The standard approach is an impression made of the implant abutment using a transfer impression coping.

There are two types of implant transfer impression coping: pick-up and re-seating copings.

The pick-up implant impression coping is used with a open faced impression tray. The tray allows access to a retaining screw that secures the impression coping to the implant. The retaining screw must extend $2-3 \mathrm{~mm}$ above the impression tray opening (Fig. 9). Impression material in injected around the impression copings first and then the tray is seated in the mouth. After the impression material has set and before removing the tray, the retaining screw is unscrewed leaving the pick-up impression coping inside the impression. The implant laboratory replica is then attached to the coping before pouring the impression with dental stone (Fig. 10). For a single tooth, the impression is made with a plastic push on coping that fits snugly over the abutment and can be picked up in a rubber base impression as above (Fig. 11).

The re-seating impression coping is used with a conventional impression tray and syringing technique and the coping remains in place on the implant after the impression material has set and the impression removed from the mouth (Fig. 12). The transfer coping is then unscrewed from the implant and attached to the laboratory replica outside the mouth and the coping/replica is re-inserted into the impression before pouring with dental stone. This technique is useful in clinical situations where there is limited space to allow for screwdrivers to undo the long retaining screws of the pick-up technique.

\section{Laboratory techniques}

Many of the laboratory techniques for implant restorations are essentially the same as for conventional prosthodontic treatment. Working casts are made using precise replicas of the abutments and machine-made gold cylinders that fit the abutments accurately can be incorporated into conventional framework wax-ups and then cast in a suitable precious gold alloy. Casts are normally made with a flexible silicone rubber gingival cuff, which is removable from the cast, allowing the dental technician to make sure that there is an accurate fit of the abutment/ restoration in the subgingival region and to help visualise and create emergence crown con-

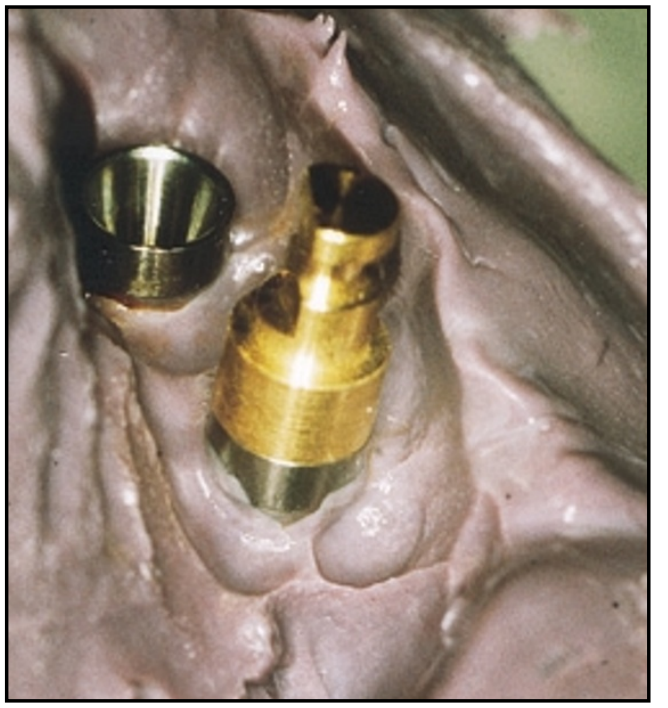

Fig. 10 The abutment replica is attached to the impression coping prior to making the cast

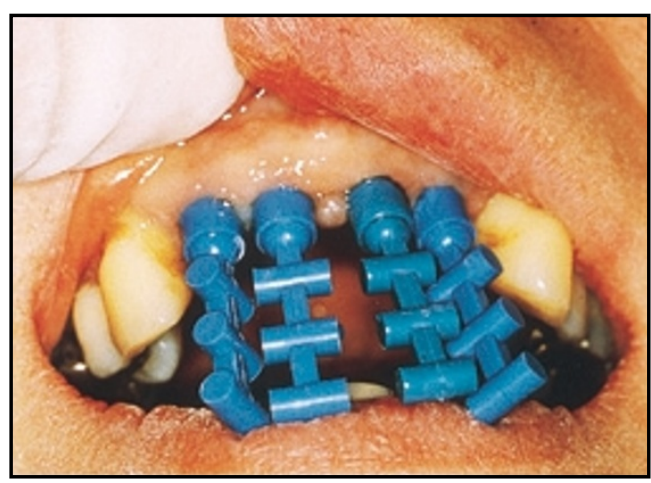

Fig. 1 1 a Branemark single tooth impression copings seated for a 'pick up' impression

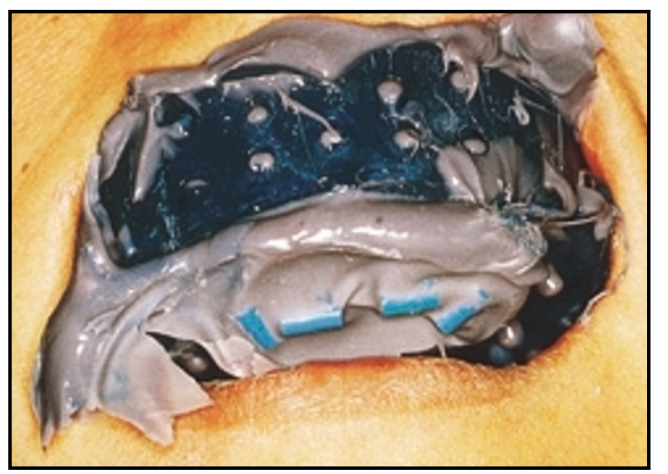

Fig. $11 \mathrm{~b}$ The copings will be removed from the mouth in the impression tours and profiles that are consistent with the gingival margin.

Implant supported bridges can either be made using conventional metal ceramic techniques or using denture teeth set in acrylic resin or using laboratory composite materials attached to a suitably designed metal framework. Choice of material will depend on a number of factors, not least restorations in the opposing dentition and any tendency towards parafunctional grinding habits.

\section{Jaw relation registration}

Conventional jaw relation records are made for single tooth and short span bridgework. The same principles for establishing occlusal plane, freeway space and recording the retruded position are applied to extensive bridgework or 


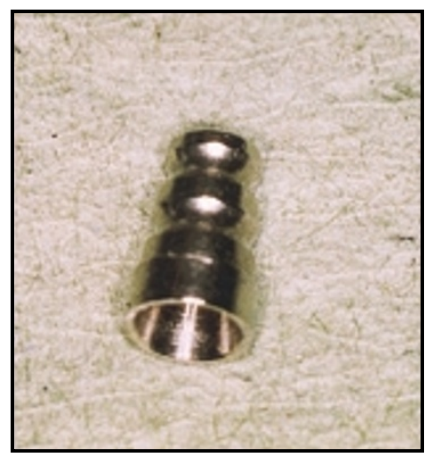

Fig. 12a Reseating impression coping. Note the ribbed design to allow for the coping to be accurately reseated in the impression

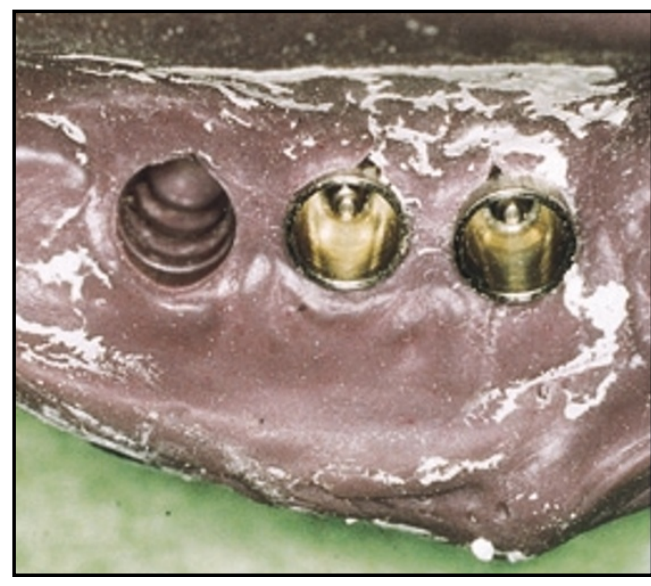

Fig. $12 \mathrm{~b}$ Impression detail. The ribbed shape can be seen in the impression

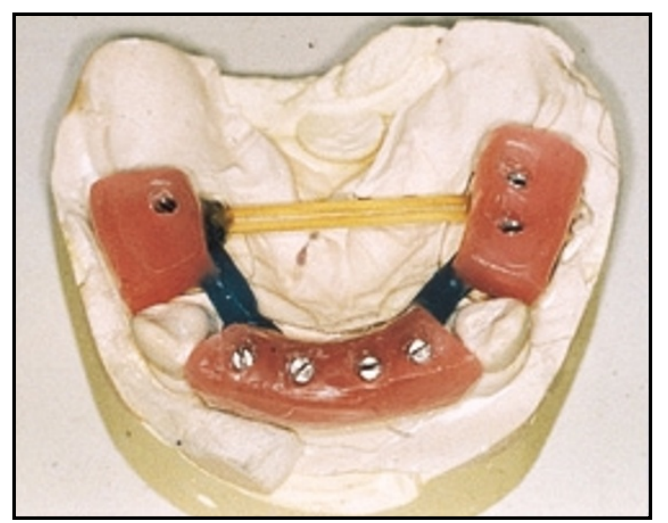

Fig. 13 Wax bite block retained onto the abutments ready to be tried in and trimmed

edentulous treatments with the advantage that bases for occlusal rims can be secured firmly to the abutments (Fig. 13).

The gold cylinders are fixed into a cold cured or light cured resin bar and a wax rim attached. This also allows the clinician to check the accuracy of the working cast and any misfit can be corrected by sectioning the acrylic bar and rejoining with an autopolymerising resin (Fig. 3).

\section{Try-in appointment}

From the records of the previous appointment the laboratory are able to fabricate a try-in using acrylic teeth set onto the resin bar or by waxing up the entire set-up (Fig. 14). This can then be used to check the appearance and occlusion as well as phonetics and cleansibility of the final restoration. It is best to check all of these features before the final framework casting is made for any longer span bridges. Single tooth restoration and short span bridges are normally produced directly from the impression stage and do not require try-in appointments.

\section{Framework try-in}

For long span bridges it is important that the final framework is tried in the mouth before the final fit appointment. It is desirable to have a perfect passive fit of the framework onto the abutments (Fig. 15). This is best checked by placing the framework in the mouth secured by only one retaining screw. It should seat fully with no obvious spring or discrepancy. This should be repeated using different abutments to ensure a perfect fit. Discrepancies in fit require sectioning of the bridge and repair with an autopolymerising resin to allow repouring of the master cast and soldering of the framework. If the framework is passive the remainder of the screws should be inserted and tightened sequentially to check that no tension is produced in the bridgework. This is usually indicated by discomfort to the patient. If the framework has been soldered it is important to repeat this appointment to check the fit once more.

\section{Restoration insertion}

If all other procedures have been performed correctly the fit appointment should be reasonably straightforward. However the application of acrylic or porcelain can produce stress within the framework which may effect fit, and the procedures for framework try-in should therefore be repeated to check the final fit. The occlusion and appearance need to be checked and the patient given appropriate oral hygiene instruction. The bridge screws can then be progressively tightened in sequence in an order that will not produce stress within the bridge.

Bridge screws are tightened to $10 \mathrm{NCm}$ and provisional restorations are placed in the screw access holes with cotton wool or gutta percha protecting the screw heads.

\section{Occlusion}

For single tooth restorations or limited span bridges, occlusal contacts need to be examined in light and heavy contact. It should be remembered that the implant is in effect ankylosed and so will not move under occlusal contact when compared with a tooth. If the restoration was made so that it was in full contact under initial occlusal contact, then once pressure was applied the implant restoration would take the full load and possibly be overloaded. In initial occlusal contact therefore the implant restoration should be in enough contact to lightly mark occlusal indicator paper but should not hold shim stock. As pressure is exerted by the patient so the implant restoration can come to hold shim stock.

It is important that the restoration is not totally out of occlusion because the risk is that there may be uncontrolled overeruption of the opposing teeth resulting in a loss of occlusal stability and occlusal interferences.

In eccentric jaw movements the ideal is not to have contact on the implant retained restoration. Where possible, immediate dis- 


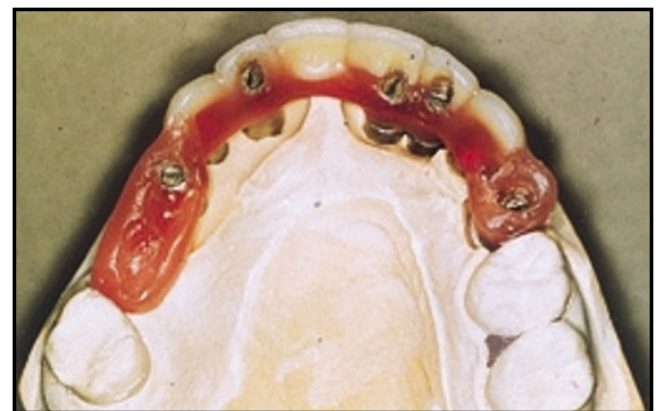

Fig. 14 Prior to casting the framework the cylinders have been linked together with a composite beam and the teeth waxed in to verify appearance and tooth position

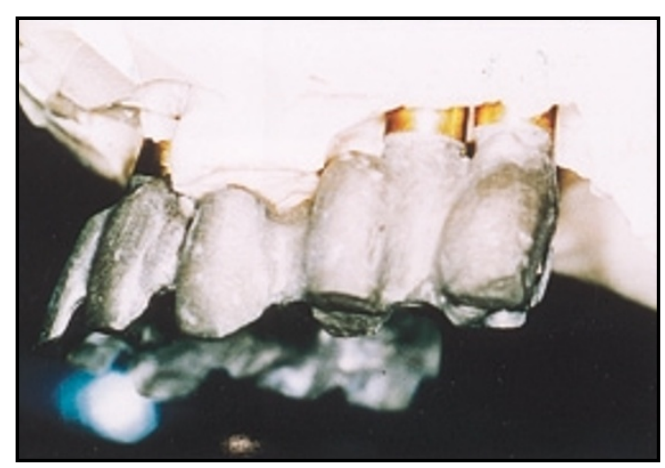

Fig. 15 The fit of the metal casting need to be verified both on the model and in the mouth

clusion on a natural canine is preferable. When anterior teeth/canines are involved in the restoration attempts should be made to provide occlusal contacts over multiple teeth and implants.

In the completely edentulous patients, implant retained dentures should follow conventional occlusal concepts of bilateral balanced or lingualised occlusion. For fixed full arch bridges the aim is simultaneous contact on anterior and posterior teeth in centric relation with anterior group function and multiple contacts in eccentric jaw movements (no canine guidance).

\section{Follow-up appointment}

At the follow-up visit the occlusion and oral hygiene should be checked. The temporary restorations over the screw holes are removed and the screws checked for tightness. Some loosening is acceptable at this stage and $1 / 4$ turn of the screw may be required to achieve full seating of the screw. If more than $1 / 4$ is required it is possible that an error in fit or occlusion is present and will require adjustment. Once happy that the screws have remained tight, the access holes can be filled with the composite resin after covering the screw head with gutta percha. Accurate long cone periapical radiographs should be taken to confirm the final fit and record the marginal bone height for future comparison.

\section{Overdentures}

Complete overdentures retained by implants are made to conventional prosthodontic principles. As with tooth borne overdentures, the bases should be designed to allow intimate tissue contact over the maximum support area and peripheral extension to achieve a border seal. Excessive reduction of the base extension in an attempt to appease patients' wishes for less tissue coverage may lead to unfavourable loading of the implants.

The main feature of the implant abutments is to provide a retentive feature for the overdenture and to improve the stability of the denture base. These might include ball, magnet or clip attachments. The main difference between implant and tooth-borne dentures is the design of the support. Cantilever support may be used to a limited degree in implant retained overdentures with a bar and clip, but not normally in tooth borne restorations.

Jaw relation records should create a freeway space of $2-4 \mathrm{~mm}$ in the incisor region and tooth position that provides for an adequate lip support and even bilateral posterior occlusal contacts which allow for freedom in movement.

\section{Conclusion}

Attention to detail during the prosthetic phase of implant treatment can produce highly aesthetic restorations which will continue to function at a high level over many years. The treatment planning phase is essential to make sure that the prosthodontic treatment is kept as straightforward as possible. Many of the shortcomings of conventional dentistry are avoided because of the use of manufactured components. However failure to observe the basic rules of conventional and implant dentistry can lead to problems which may be difficult and expensive to overcome. 